\title{
Synthèse des caractéristiques physico-chimiques et effets écotoxicologiques du Tihan 175 O-TEQ utilisé dans la protection phytosanitaire du cotonnier au Bénin
}

\author{
Prudencio T. AGBOHESSI ${ }^{1,2 *}$, Ibrahim IMOROU TOKO ${ }^{2}$, Eloi Y. ATTAKPA ${ }^{2}$ et \\ Patrick KESTEMONT ${ }^{1}$
}

${ }^{1}$ Université de Namur, Faculté des Sciences, Unité de Recherche en Biologie Environnementale et Evolutive (URBE), 61 rue de Bruxelles, 5000 Namur, Belgique.

${ }^{2}$ Université de Parakou, Faculté d'Agronomie, Département de Production Animale, Unité de Recherche en Aquaculture et en Ecotoxicologie Aquatique (URAEAq), BP 123, Parakou, Bénin.

*Auteur correspondant; E-mail : agbohessiprudencio@yahoo.fr; Tél :0022997645416

\section{RESUME}

La culture du coton au Bénin au stade actuel des pratiques est très consommatrice de pesticides. Parmi ceux recommandés dans le traitement phytosanitaire du cotonnier, il y a le Tihan 175 O-TEQ utilisé en alternatif à l'endosulfan et efficace particulièrement contre le carpophage Helicoverpa armigera, l'acarien Polyphagotarsonemus latus, le phyllophage Sylepta derogata et Aphis gossipii. Le Tihan 175 O-TEQ est constitué du flubendiamide $(100 \mathrm{~g} / \mathrm{l})$ et du spirotétramate $(75 \mathrm{~g} / \mathrm{l})$ et utilisé pendant 2 à 4 traitements sur les 6 recommandés en fonction de la zone écologique. Ces deux matières actives introduites en 2007 sont encore très mal connues. Un travail de synthèse nous a permis d'établir quelques propriétés physico-chimiques et écotoxicologiques de ces produits chimiques. Ainsi, le flubendiamide lipophile a un potentiel de bioconcentration et de bioaccumulation dans le foie et les tissus graisseux des animaux. Il agit sur la croissance et la reproduction en provoquant chez les rats par exemple, un retard dans l'apparition du sexe chez les mâles F1 et une augmentation du poids des ovaires et de l'utérus chez les femelles F0. Les individus femelles ont une grande sensibilité au flubendiamide par rapport aux mâles. Le spirotétramate hydrosoluble a des effets nocifs sur la croissance, la survie des alevins, les testicules et les spermatozoïdes des rats. Il provoque également une réduction de la production d'œufs, du taux d'éclosion et du poids corporel des portées chez Anas platyrhynchos. On a constaté aussi, une réduction du pourcentage d'œufs viables ou d'œufs pondus chez Colinus virginianus.

(C) 2012 International Formulae Group. All rights reserved.

Mots clés: Pesticides, Tihan 175 O TEQ, flubendiamide, spirotétramate.

\section{INTRODUCTION}

Au Bénin (Figure 1), comme dans la plupart des pays de l'Afrique de l'Ouest francophone (Burkina Faso, Mali, Niger, etc), l'agriculture occupe une place prépondérante dans les activités socioéconomiques. Elle concerne plus de $70 \%$ de la population active, contribue à $40 \%$ du Produit Intérieur Brut (PIB) et assure plus de $80 \%$ des recettes d'exportation (AIC, 2008). L'analyse économique a révélé que depuis 1992, c'est la 


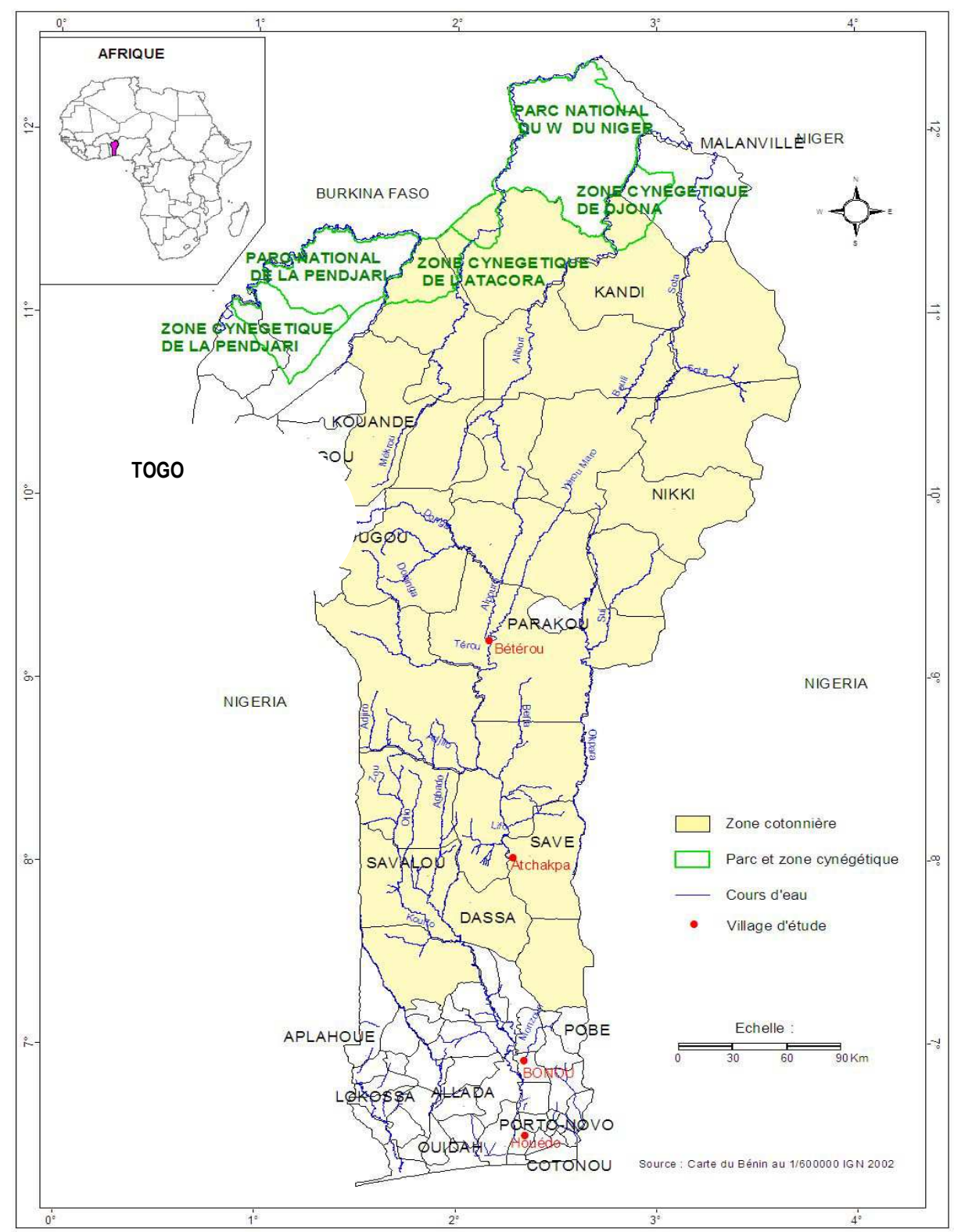

Figure 1: Carte administrative du Bénin avec les zones cotonnières. 
filière coton qui domine avec à elle seule, plus de $74 \%$ des exportations (MEF, 2010). En 2008, cette filière représentait environ $90 \%$ des recettes agricoles et $45 \%$ des rentrées fiscales (AIC, 2009). Plus de 52\% de la population rurale tirent des ressources de la production cotonnière (Gomgnimbou et al., 2009). Au Bénin, le coton constitue donc «le moteur blanc du développement» mais en même temps le cotonnier est considéré comme «une culture dévastatrice de l'environnement » (Ton, 1995).

En effet, la production agricole en Afrique de l'Ouest en général, et au Bénin en particulier, se caractérise par l'utilisation d'engrais minéraux pour la fertilisation des sols et de pesticides chimiques pour la lutte contre les adventices et le traitement phytosanitaire des plantes. Cette culture utilise à elle seule près de $96 \%$ des engrais chimiques employés au Bénin (IFDC, 2005) et représente aussi près de $90 \%$ du marché des insecticides en Afrique de l'ouest francophone (MAEP, 2005). Mais, seuls $0,1 \%$ de ces pesticides pulvérisés dans les champs atteignent leur cible, le reste se répartit dans les écosystèmes pour contaminer la terre, l'eau et l'air (Primentel et Levitain, 1986). La fraction émise dans l'atmosphère par volatilisation finit aussi par regagner, sous forme de retombées atmosphériques, les sols et les eaux de surface lors des précipitations (Haraguchi et al., 1994). Ces produits dangereux affectent l'équilibre des écosystèmes et conduisent même parfois à l'extinction de certaines espèces (Adégbidi, 2000 ; Fadoégnon et al., 2006). Au nombre des pesticides utilisés au Bénin, il y a le Tihan 175 O-TEQ dont les effets écotoxicologiques sont peu connus. Cet article vise donc à faire le bilan à travers une synthèse documentaire, des caractéristiques physico-chimiques et effets toxicologiques potentiels des principales matières actives du Tihan sur les organismes animaux aussi bien terrestres qu'aquatiques.
Historique et programme de traitement phytosanitaire du cotonnier au Benin

La culture du coton exige des programmes de traitement phytosanitaire car elle paie un lourd tribut aux ravageurs. Les pertes sont énormes et varient selon le nombre de saison de pluie de la zone écologique, entre 20 et $60 \%$ du potentiel de production de la plante (Deguine et al., 1993 ; Follin et Deat, 1999). En dehors du traitement biologique dont le taux d'adoption reste encore très faible en raison des rendements bas, deux types de traitement chimique sont vulgarisés pour lutter contre ces ravageurs: la Lutte Etagée Ciblée (LEC) introduite au Bénin depuis 1988 (Totin, 2004) et le traitement classique calendaire qui reste le plus répandu.

La LEC est une technique pour laquelle les traitements à appliquer dépendent des parasites dont le seuil de nuisance est observé sur la parcelle. Elle permet de réduire de près de $50 \%$ les quantités d'insecticides utilisés pour la lutte classique (Deguine et al., 1993 ; Ferron et al., 2006).

Jusqu'à la campagne cotonnière 19981999, ces programmes de traitement chimique étaient entièrement basés sur l'utilisation des matières actives de la famille des pyréthrinoïdes, solution idéale pour remplacer les organochlorés jugés alors trop toxiques (Katary, 2003). Mais, face aux chutes drastiques des rendements, provoquées par la résistance du plus redoutable ennemi du cotonnier (Helicoverpa armigera) aux pyréthrinoïdes, d'autres programmes de lutte chimique ont été mis en œuvre à partir de la campagne 1999-2000 dans lesquels les organochlorés ont été réintroduits à travers l'endosulfan en remplacement des pyréthrinoïdes (Katary, 2003). Mais, en février 2007, suite aux nuisances de l'endosulfan sur l'environnement et les populations humaines (décès de plusieurs personnes dus à l'intoxication à l'endosulfan) (AIC, 2009), cet insecticide a été officiellement interdit et remplacé par le Tihan 175 O-TEQ (Mbaye, 2008). 
Au-delà des difficultés liées à l'approvisionnement en intrants pour la campagne agricole 2012-2013, les programmes de traitement phytosanitaire classique actuellement en vigueur dans toutes les zones cotonnières du Bénin sont consignés dans le Tableau 1 (zone nord, zone centre et sud). De l'analyse de ce tableau, il ressort que 6 traitements au minimum sont recommandés. Le $1^{\text {er }}$ intervient au $50^{\text {ème }}$ jour (j) après semis et 2 traitements successifs doivent être séparés de 14 j (Katary, 2003). Ensuite, ces programmes de traitement phytosanitaire du cotonnier sont divisés en 3 fenêtres : La $11^{\text {ère }}$ est constituée du Tihan 175 O-TEQ formulé pour le traitement TBV (Très Bas Volume), utilisé à une dose de $0,21 /$ ha au $1^{\text {er }}, 2^{\text {ème }}, 4^{\text {ème }}$ et $5^{\text {ème }}$ traitement dans le département de l'Alibori et la commune de Kérou et au $1^{\text {er }}$ et $2^{\text {ème }}$ traitement dans le reste des zones de production cotonnière (Figure 1). Le Tihan 175 O-TEQ est efficace sur les chenilles de la capsule, particulièrement $H$. armigera, l'acarien Polyphagotarsonemus latus, le phyllophage Sylepta derogata et Aphis gossipii (AIC, 2009). La $2^{\text {ème }}$ fenêtre est constituée d'un binaire acaricide à dose faible à moyenne d'organophosphorés $(0,2$ à 2 1/ha dans la zone nord) et à forte dose d'organophosphorés ( 1 à 2 1/ha dans le centre et le sud du pays), tous formulés $\mathrm{CE}$ (Concentrés Emulsifiables). Les binaires acaricides à dose faible à moyenne d'organophosphorés contiennent un pyréthrinoïde associé à un organophosphoré acaricide faible voire moyenne dose (Tableau 2). Les binaires acaricides à doses fortes d'organophosphorés contiennent aussi un pyréthrinoïde associé à un organophosphoré acaricide mais à forte dose (Tableau 2). L'association pyréthrinoïde-organophosphoré acaricide est recommandée pour lutter contre les infestations simultanées de tarsonèmes $P$. latus, de carpophages $H$. armigera et de Diparopsis $s p$ et de phyllophages $S$. derogata (Katary, 2003). La $3^{\text {ème }}$ fenêtre est constituée de produits binaires aphicides en formulation CE ou O-TEQ (Oil Toxicity EQuivalence) pour le traitement TBV, contenant un pyréthrinoïde associé à un néonicotinoïde (Tableau 2). L'association pyréthrinoïdesaphicide en fin de cycle vise à lutter, dans les départements du Borgou, de l'Atacora et de la Donga, contre les carpophages et les pucerons (Katary, 2003).

\section{Caracteristiques physico-chimiques et effets ecotoxicologiques potentiels du flubendiamide et du spirotetramate}

Le Tihan 175 O-TEQ utilisé au Bénin est un binaire constitué de flubendiamide (100 $\mathrm{g} / \mathrm{l})$ et de spirotétramate $(75 \mathrm{~g} / \mathrm{l})$. Ce sont de nouvelles molécules en utilisation dans plusieurs pays du monde. Au Bénin, ces molécules sont recommandées pour 2 à 4 traitements sur 6 pour lutter singulièrement contre l'H. armigera, le plus redoutable ennemi du cotonnier (AIC, 2009).

\section{Le flubendiamide \\ Caractéristiques physico-chimiques}

Le flubendiamide ou $\mathrm{N}^{2}$-[1,1-dimethyl2-(methylsulfonyl)ethyl]-3-iodo- $\mathrm{N}^{1}$-[2-

methyl- = 4-[1,2,2,2-tetrafluoro-1(trifluoromethyl) ethyl]phenyl]-1, 2benzenedicarboxamide (Figure 2a) est la première matière active faisant partie du groupe des diamides de l'acide phtalique ou des benzenedicarboxiamides (Sattelle et al., 2008). Il agit chez les insectes cibles sur le récepteur ryanodine, un canal de sortie de calcium $\left(\mathrm{Ca}^{2+}\right)$ impliqué dans la contraction du muscle (Ebbinghaus et al., 2007 ; Masaki et al., 2006). Le flubendiamide a une demi-vie dans le sol de 33,6 à 34,9 j (Tableau 3). La photodégradation sur les surfaces de sol est une voie de dissipation importante du flubendiamide dans l'environnement. La demi-vie du flubendiamide soumis à la phototransformation solaire est en moyenne de $15 \mathrm{j}$ en Europe centrale (APVMA, 2009). Le $1^{\text {er }}$ produit de la photodégradation du flubendiamide dans le sol est le des-iodoflubendiamide (Figure 2b) qui est beaucoup plus stable que le composé d'origine avec une demi-vie > 1 an quel que soit le lieu et la 
saison (Ebbinghaus et al., 2007 ). Toutefois, certaines chaînes de cette molécule (le desiodo-flubendiamide) peuvent se briser au cours de cette dégradation pour donner de l'acide oxalinique du flubendiamide ou se minéraliser en dioxyde de carbone. Par contre, le flubendiamide est stable en condition anaérobique en absence de lumière et à $\mathrm{pH}$ légèrement acide. Son coefficient d'adsorption élevé (1076-3318 1/kg) indique la possibilité d'une forte agglutination de celui-ci après son épandage dans les champs (Justus et al., 2007). Dans l'eau, le flubendiamide a une demi-vie de 15 à $39 \mathrm{j}$. Les produits de la phototransformation dans l'eau sont le desiodo-flubendiamide (maximum 10,1 à 31,9\% après $168 \mathrm{~h}$ ), le 3-hydroxyflubendiamide (Figure 2c) (0,2 à $13 \%$ entre 24 et 168 h) et le 3-hydroxy-perfluoroalkyl-flubendiamide $(0,2$ à $13 \%$ entre 48 et 168 h) (APVMA, 2009). Dans le système eau/sédiment, la dissipation est la principale voie de transfert. En effet, lorsque le flubendiamide est lessivé vers les eaux à un temps $\mathrm{t}_{0}, 0,64$ à $68 \%$ est dans la colonne de l'eau et 24 à $29 \%$ se dépose directement dans le sédiment. $125 \mathrm{j}$ après, il ne reste que 3 à $4 \%$ dans la colonne d'eau mais 73 à $75 \%$ sont déjà dans le sédiment (APVMA, 2009). La pression de vapeur (< $10^{-4} \mathrm{~Pa}$ à $20{ }^{\circ} \mathrm{C}$ ) et la constance de la loi d'Henry $\left(2.10^{-9} \mathrm{~Pa} . \mathrm{m}^{3} / \mathrm{mol}\right) \mathrm{du}$ flubendiamide justifient sa faible volatilité dans l'environnement et il ne devrait pas se volatiliser à partir des surfaces de l'eau ou de la surface des sols humides. Par conséquent, on ne s'attend pas à trouver des résidus de flubendiamide dans l'air, ou encore que ceuxci fassent l'objet d'un transport à grande distance. Il a aussi une solubilité faible dans l'eau $\left(0,029 \mathrm{mg} / 1\right.$ à $\left.20^{\circ} \mathrm{C}\right)$ (Tohnishi et al., $2005)$ et une lipophilie élevée $(\log \mathrm{P}=4,1$ à pH 4 - 10 à $20{ }^{\circ} \mathrm{C}$ ) (Hall, 2007). Il y a donc une évidence pour son accumulation dans le foie et la graisse des animaux. Le flubendiamide s'hydrolyse très légèrement à pH 4 à 9 avec à la fin plus de $93 \%$ du produit de départ (APVMA, 2009).

\section{Effets sur les organismes animaux}

Le flubendiamide serait un perturbateur endocrinien et aurait des effets néfastes sur le développement chez l'homme. Des études mentionnent que lorsque le flubendiamide est administré par voie orale, 93 à $99 \%$ sont excrétés via les fèces $(75 \%$ après $24 \mathrm{~h}$ et > $90 \%$ après 48 h) (APVMA, 2009). Les rats femelles ont tendance à accumuler beaucoup plus le flubendiamide dans leurs tissus graisseux et à les excréter via les fèces plus lentement que les mâles. Une étude sur les chèvres allaitantes exposées à 176 et 370 $\mathrm{mg} / \mathrm{kg}$ à travers l'alimentation pendant 4 jours consécutifs a montré $5 \mathrm{~h}$ après la prise de la dernière dose que 25 à $45 \%$ sont excrétés (24 à $44 \%$ via les fèces, 0,25 à $0,5 \%$ via l'urine, 0,4 à $0,5 \%$ via le lait) et il a été noté une faible accumulation de résidus dans le lait, le muscle et les reins mais une forte accumulation dans le foie et la graisse (Justus et al., 2007). Les mêmes auteurs ont rapporté chez des poules pondeuses exposées à 9 et $17 \mathrm{mg} / \mathrm{kg}$ d'aliment pendant $14 \mathrm{j}$, que l'excrétion a commencé aussitôt après la $1^{\text {ère }}$ administration (62 à $66 \%$ de la radioactivité) et que les poules sacrifiées après $24 \mathrm{~h}$ de la fin de l'exposition ont présenté une accumulation de résidu de $24 \%$ dans les muscles et 5 à $8 \%$ dans les œufs mais la concentration de la radioactivité est faible dans les muscles et très élevée dans les œufs, le foie et la graisse. Par ailleurs, le flubendiamide n'est ni génotoxique, ni carcinogène, ni neurotoxique (APVMA, 2009). Les études sur la reproduction du rat ont montré des effets nocifs du flubendiamide sur la progéniture de celui-ci. Hall (2007) a rapporté chez des rats adultes exposés à 2000 et $20000 \mathrm{mg} / \mathrm{kg}$ (voie orale) pendant 16 semaines, un retard dans l'apparition du sexe chez les mâles F1, une coloration sombre du foie chez les femelles F0 et une augmentation du poids des ovaires et de l'utérus. Le même auteur a observé chez des rats exposés par gavage à une dose de $2000 \mathrm{mg} / \mathrm{kg}$, un retard dans la séparation préputiale, et une augmentation du globe oculaire chez les mâles F1, une augmentation des reins, une 
hépatotoxicité et une augmentation du poids pituitaire chez les femelles F1. En outre, aucune toxicité aiguë n'a été observée au niveau des poissons d'eau douce à la limite de la solubilité dans l'eau (29,9 $\mu \mathrm{g} / \mathrm{l})$ (Hall, 2007). Cependant, des investigations effectuées sur Lepomis macrochirus ont révélé un léger potentiel de bioconcentration $(\mathrm{BCF}=$ 73 ) et de biotransformation du flubendiamide et de son métabolite le des-iodoflubendiamide (APVMA, 2009). Des études de toxicité sur Pimephales promelas ont donné des "No Observed Effect Concentration (NOEC)', (21 jours) de 0,06 $\mathrm{mg} / \mathrm{l}$ et des NOEC (survie, croissance) de 60,2 $\mu \mathrm{g} / 1$ (APVMA, 2009). Plusieurs études de toxicité aiguë menées par Hall (2007) en conditions statiques ont donné chez Lepomis macrochirus une Concentration Létale à 50\% CL50-96 $\mathrm{h}>67,7 \mu \mathrm{g}$ de matière active (m.a.)/l, chez Cyprinus carpio une CL50-96 h $>84,7 \mu \mathrm{g}$ m.a./l, chez Pimephales promelas une CL50-96 $\mathrm{h}>66,5 \mu \mathrm{g}$ m.a./l et chez Oncorhynchus mykiss une CL50-96 h > 60,0 $\mu \mathrm{g}$ m.a./l.

\section{Le spirotetramate \\ Caractéristiques physico-chimiques}

Le spirotétramate ou carbonate d'éthyle et de cis-3-(2,5-diméthylphényl)-8-méthoxy2-oxo-1-azaspiro [4.5] déc-3-én-4-yle (Figure 2d) est un insecticide systémique à spectre limité classé dans la catégorie des dérivées de l'acide tétramique (Bruck et al., 2009). C'est la toute nouvelle classe d'insecticide dont l'action est d'inhiber la biosynthèse des lipides chez les insectes cibles (Nauen et al., 2006). Ces propriétés physico-chimiques sont résumées dans le Tableau 4 . Il est stable et ne se bioaccumulerait pas. En milieu terrestre, la principale voie de transformation du spirotétramate est la biotransformation. Le spirotétramate n'est pas persistant dans le sol et se dissipe en moins d'une journée en conditions de laboratoire et de terrain (PRD, 2008-07). Les principaux produits de transformation générés par la biotransformation du spirotétramate dans le sol sont le spirotétramate-énol (Figure 2e) et le céto-hydroxy-spirotétramate (Figure 2f). En conditions de laboratoire, la demi-vie pour ces transformations est de 3,3 à $12,3 \mathrm{j}$ et de 1,5 à 16,7 j, respectivement (Bruck et al., 2009). La phototransformation sur le sol ne devrait pas constituer une voie de transformation importante pour le spirotétramate. Dans une étude en laboratoire, on a constaté que les principaux produits de transformation étaient le spirotétramate-énol, le céto-hydroxyspirotétramate et l'acide diméthylbenzoïque (PRD, 2008-07). L'acide diméthylbenzoïque se forme exclusivement par phototransformation, et il ne devrait pas être présent en concentrations élevées dans l'environnement (Bruck et al., 2009). En outre, la persistance de l'acide diméthylbenzoïque ne devrait pas soulever de préoccupations compte tenu de la structure relativement simple de ce composé (PRD, 2008-07). Le spirotétramate est soluble dans l'eau $(29,9 \mathrm{mg} / \mathrm{l})$ et ne se liera pas fortement aux particules de sol. De telles caractéristiques chez un composé accroissent sa possibilité d'être lessivé. Le spirotétramate n'est pas persistant aussi dans l'eau, où il se transforme en une journée environ par biotransformation (PRD, 2008-07). Dans les études de laboratoire, les principaux produits de transformation générés par la biotransformation du spirotétramate en milieu aquatique aérobie sont le spirotétramate-énol et le céto-hydroxy-spirotétramate. En conditions aérobies, le spirotétramate-énol s'est montré plus persistant que le composé d'origine (demi-vie se situant entre 38 et $59 \mathrm{j}$ ), tandis que le céto-hydroxy-spirotétramate est stable (PRD, 2008-07). Le spirotétramate s'hydrolyse pour donner du spirotétramateénol, lequel résiste à l'hydrolyse. La phototransformation pourrait aussi constituer une voie de dissipation du spirotétramate en milieu alcalin. D'après les résultats issus de l'étude sur la phototransformation dans de l'eau naturelle stérilisée (à pH 7,9), la demivie pour la phototransformation nette est de $0,22 \mathrm{j}$. Les principaux produits de 
transformation caractérisés dans le cadre de cette étude sont le spirotétramate-énol, le méthoxy-cyclohexanone et l'acide méthoxycyclohexyl-amino-carboxylique (Bruck et al., 2009). La pression de vapeur $\left(5,6.10^{-9} \mathrm{~Pa}\right.$ à 20 $\left.{ }^{\circ} \mathrm{C}\right)$ et la constante de la loi d'Henry $(6,99.10$ $\left.{ }^{8} \mathrm{~Pa} . \mathrm{m}^{3} / \mathrm{mol}\right) \mathrm{du}$ spirotétramate indiquent que ce produit n'est pas volatile dans l'environnement et qu'il ne devrait pas se volatiliser à partir des surfaces de l'eau ou de la surface des sols humides. Par conséquent, on ne s'attend pas à trouver des résidus de spirotétramate dans l'air, ou encore que ceuxci fassent l'objet d'un transport à grande distance (PRD, 2008-07).

\section{Effets sur les organismes animaux}

Selon l'étude 2005- 1314138 de l'Agence de Réglementation de la Lutte Antiparasitaire (ARLA, 2005), le comportement pharmacocinétique du spirotétramate se caractérise chez le rat par une absorption et une élimination plasmatique rapide. Le produit était dans une large mesure absorbé, 89 à $98 \%$ de la radioactivité récupérée au total ayant été excrétés par voie rénale. L'analyse quantitative par autoradiographie du corps entier a permis de constater que les plus fortes concentrations de la substance et de ses métabolites se trouvaient dans le foie, les reins, le tractus gastro-intestinal, la vessie et le sang (ARLA, 2005). L'étude menée dans le cadre de l'évaluation toxicologique du spirotétramate indique qu'il ne s'est montré ni cancérogène, ni génotoxique. Ce produit n'était pas non plus tératogène chez le lapin (PRD, 2008). Chez le rat, la tératogénicité ne se manifestait qu'à des doses toxiques pour les mères (ARLA, 2005). On a noté une variabilité de la réponse toxicologique selon les espèces soumises aux essais, variabilité qui pourrait refléter des différences de métabolisation. Les souris se sont montrées relativement insensibles à la substance à l'essai; par contre, des effets nocifs ont été notés chez les rats et les chiens. Chez le rat, les mâles ont semblé plus sensibles aux effets toxiques du spirotétramate que les femelles; il a été établi que le système reproducteur mâle (spermatozoïdes, testicules et épididymes) était une cible de la toxicité. Des effets toxiques sur les spermatozoïdes ont été enregistrés chez les mâles de la génération F1 à des doses plus faibles que chez les mâles de la génération parentale (ARLA, 2005). Il semblerait que l'espèce la plus sensible soit le chien, ceci d'après les effets constatés sur le thymus et le système nerveux central et d'après les perturbations des hormones thyroïdiennes observées. Une diminution des taux d'hormones thyroïdiennes a invariablement été relevée dans toutes les études sur le chien (ARLA, 2004). On a noté des signes cliniques de neurotoxicité ainsi qu'une pathologie cérébrale chez le chien (PRD, 2008). La même étude a fait état chez Anas platyrhynchos de la réduction de la production d'œufs, du taux d'éclosion et du poids corporel des petits (Concentration Sans Effet Observé CSEO $=28,8 \mathrm{mg} \mathrm{m}$.a. $/ \mathrm{kg}$ d'aliment) et chez Colinus virginianus de la réduction $\mathrm{du}$ poids corporel moyen à l'éclosion et du pourcentage d'embryons viables/œufs pondus (CSEO = $264 \mathrm{mg}$ m.a. /kg d'aliment). Dans des études à long terme, on a observé chez Daphnia magna une diminution du nombre de descendants par parent (ARLA, 2006e). La même étude a noté une inhibition de la formation des coquilles chez les huitres. Dans un essai de toxicité aux premiers stades vitaux sur Pimephales promelas, on a vu que le spirotétramate avait des effets nocifs sur le taux d'éclosion (CSEO $33 \mathrm{j}=1,16 \mathrm{mg} \mathrm{m} . \mathrm{a} . / \mathrm{l})$, la survie des alevins (CSEO $33 \mathrm{j}=0,534 \mathrm{mg} \mathrm{m} . \mathrm{a} . / \mathrm{l})$ et la croissance (1,16 mg m.a./l) (ARLA, 2006d). L'étude de sa toxicité aiguë a donné chez Oncorhynchus mykiss une $\mathrm{CL}_{50}-96 \mathrm{~h}=2,54$ mg m.a. /l et une CSEO de $0,825 \mathrm{mg}$ m.a. $/ 1$ (ARLA, 2006c), chez Cyprinodon carpio une $\mathrm{CL}_{50}-96 \mathrm{~h}=2,59 \mathrm{mg}$ m.a./1 (ARLA, 2006a) et chez Lepomis macrochirus une $\mathrm{CL}_{50}-96 \mathrm{~h}=$ 2,2 mg m.a. /l (ARLA, 2006b). 


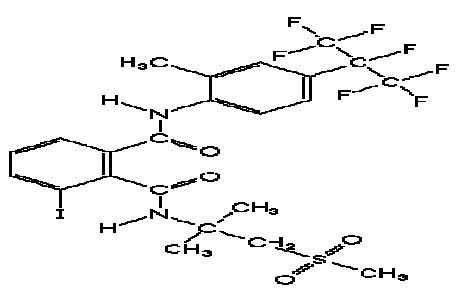

a) Flubendiamide

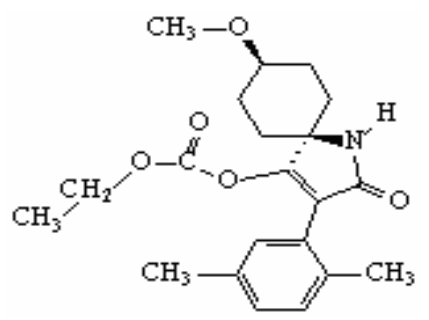

d) Spirotétramate

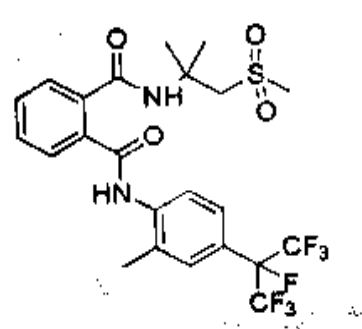

b) Des-iodoflubendiamide

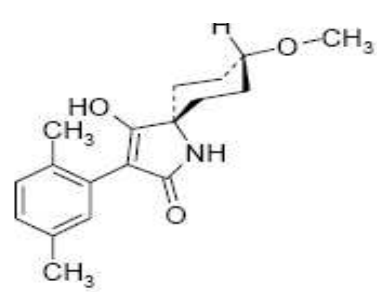

e) Spirotétramate-énol

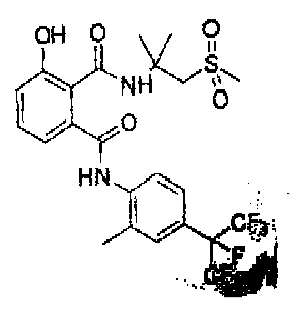

c) 3-Hydroxyflubendiamide

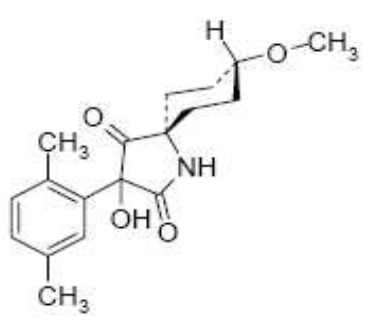

f) Cétohydroxyspirotétramate

Figure 2: Formules chimiques du flubendiamide, du spirotétramate et de quelques produits de leur dégradation. 
Tableau 1: Programmes de traitement phytosanitaire classique du cotonnier.

\begin{tabular}{|c|c|c|c|c|c|c|}
\hline \multirow[b]{2}{*}{ Produits } & \multicolumn{2}{|c|}{$\begin{array}{l}\text { L'Alibori et Kérou } \\
\text { dans l'Atacora }\end{array}$} & \multicolumn{2}{|c|}{$\begin{array}{l}\text { Le Borgou et le reste de } \\
\text { l'Atacora et la Donga }\end{array}$} & \multicolumn{2}{|c|}{ Zone Centre et Sud } \\
\hline & $\begin{array}{l}\text { Dose } \\
(\mathbf{l} / \mathbf{h a})\end{array}$ & $\begin{array}{l}\text { Utilisation } \\
\text { (traitement) }\end{array}$ & $\begin{array}{l}\text { Dose } \\
\text { (1/ha) }\end{array}$ & $\begin{array}{c}\text { Utilisation } \\
\text { (traitement) }\end{array}$ & $\begin{array}{l}\text { Dose } \\
\text { (l/ha) }\end{array}$ & $\begin{array}{c}\text { Utilisation } \\
\text { (traitement) }\end{array}$ \\
\hline $\begin{array}{l}\text { Tihan } 175 \text { O- } \\
\text { TEQ }\end{array}$ & 0,2 & $1^{\mathrm{er}}, 2^{\mathrm{e}}, 4^{\mathrm{e}}$, et & 0,2 & $1^{\text {er }}$ et $2^{\mathrm{e}}$ & 0,2 & $1^{\mathrm{er}}$ et $2^{\mathrm{e}}$ \\
\hline $\begin{array}{l}\text { Binaires } \\
\text { acaricides }\end{array}$ & $0,2-2$ & $3^{\mathrm{e}}$ & $0,6-2$ & $3^{\grave{\mathrm{e}}}$ et $4^{\grave{\mathrm{e}}}$ & $1-2$ & $3^{\grave{e ̀ ~}}$ et $4^{\grave{e}}$ \\
\hline $\begin{array}{l}\text { Binaires } \\
\text { aphicides }\end{array}$ & & & 1 & $5^{\text {è }}$ et $6^{\text {è }}$ & 1 & $5^{\grave{e ̀ ~}}$ et $6^{\text {è }}$ \\
\hline $\begin{array}{l}\text { Défoliant } \\
\text { Havarde } 25 \mathrm{~F}\end{array}$ & 2 & $6^{\text {è }}$ & 0,2 & $\begin{array}{c}\text { En remplacement } \\
\text { au choix du } 6^{\text {è }} \\
\text { traitement } \\
\text { uniquement }\end{array}$ & & \\
\hline
\end{tabular}

Source : AIC (2009).

Tableau 2: Liste des produits phytosanitaires recommandés au Bénin.

\begin{tabular}{lcc}
\hline Produits & Noms commerciaux & Matières actives et concentrations \\
\hline Tihan 175 0-TEQ & Tihan 175 0-TEQ & Flubendiamide-spirotétramate 100-75 g/l \\
\hline & Alphacal P 218 CE & Alphacyperméthrine-profenofos 18-200 g/l \\
& Dursban B 218 CE & Cyfluthrine-chlorpyrifos-éthyl 18-200 g/l \\
& Cyflutralm P 218 CE & Cyfluthrine-profenofos 18-200 g/l \\
& Cyclofos 236 CE & Cyperméthrine-chlorpyrifos-éthyl 36-200 g/l \\
& Nurelle D 236 CE & Cyperméthrine-chlorpyrifos-éthyl 36-200 g/l \\
Binaires acaricides à & Sherpos 180 CE & Cyperméthrine-triazophos 30-150 g/l \\
dose faible à & Duo 448 CE & Cyperméthrine H.C. - profenofos 48-400 g/l \\
moyenne & Décis T 160 CE & Deltaméthrine-triazophos 10-150 g/l \\
d'organophosphorés & Cotalm P 218 CE & Lambdacyhalothrine-profenofos 18-200 g/l \\
& Lambdacal P 215 CE & Lambdacyhalothrine-profenofos 15-200 g/l \\
& Amiral 430 EC & Lambdacyhalothrine-profenofos 30-400 g/l \\
& Fury P 215 CE & Zétacyperméthrine profenofos 15-200 g/l \\
& Pyrinex Quick 212 EC & Deltaméthine-chlorpyrifos-éthyl 12-200 g/l \\
& Lambdacal P 645 EC & Lambdacyhalothrine-profenofos 45-600 g/l \\
\hline \multirow{4}{*}{ Binaires acaricides à } & Alphacal P 318 CE & Alphacyperméthrine-profenofos 18-300 g/l \\
dose forte & Dursban B 318 CE & Cyfluthrine-chlorpyrifos-éthyl 18-300 g/l \\
d'organophosphorés & Cyflutralm P 318 CE & Cyfluthrine-profenofos 18-300 g/l \\
& Cyclofos 335 CE & Cyperméthrine-chlorpyrifos-éthyl 35-300 g/l \\
& Nurelle D 335 CE & Cyperméthrine-chlorpyrifos-éthyl 35-300 g/l \\
& Duo 648 CE & Cyperméthrine H.C. - profenofos 48-600 g/l \\
& Décis T 258,75 CE & Deltaméthine-triazophos 18,75-250 g/l \\
& Lambdacal P 315 CE & Lambdacyhalothrine-profenofos 15-300 g/l \\
& Amiral 630 CE & Lambdacyhalothrine-profenofos 30-600 g/l \\
& &
\end{tabular}


Fury P 315 CE

Sherphos $280 \mathrm{CE}$

Lambdacal P 630 CE
Zétacyperméthrine profenofos $15-300 \mathrm{~g} / 1$

Cyperméthrine-triazophos 30-250 g/l

Lambdacyhalothrine-profenofos 30-600 g/l

\begin{tabular}{lcc}
\hline & Conquest C 88 CE & Cyperméthrine-acétamipride 72-16 g/l \\
Capt 88 CE & Cyperméthrine-acétamipride 72-16 g/l \\
Binaires aphicides & Emir 88 CE & Cyperméthrine-acétamipride 72-16 g/l \\
& Thunder 145 OD & Bêtacyfluthrine-imidaclopride 45-100 g/1 \\
\hline Défoliant & Havarde 25F & Diméthipin 25 F \\
\hline Source : AIC (2009). & &
\end{tabular}

Tableau 3: Synthèse des caractéristiques physico-chimiques du flubendiamide.

\begin{tabular}{|c|c|c|}
\hline & Flubendiamide & Références \\
\hline Familles & Diamide de l'acide phtalique & $\begin{array}{c}\text { Ebbinghaus et al., } 2007 \\
\text { Sattelle et al., } 2008 \\
\end{array}$ \\
\hline Formule brute & $\mathrm{C}_{23} \mathrm{H}_{22} \mathrm{~F}_{7} \mathrm{IN}_{2} \mathrm{O}_{4} \mathrm{~S}$ & APVMA, 2009 \\
\hline $\begin{array}{l}\text { Mode d'action sur } \\
\text { les insectes ciblés }\end{array}$ & Agit sur le récepteur ryanodine & $\begin{array}{c}\text { Ebbinghaus-Kintscher et al., } \\
2006 \text {; Masaki et al., } 2007\end{array}$ \\
\hline Volatilité & faible & APVMA, 2009 \\
\hline Hydro-solubilité & $\begin{array}{c}29,9 \pm 2,87 \mu \mathrm{g} / \mathrm{l} \text { à } \mathrm{pH} 4-10 \text { à } 20 \\
{ }^{\circ} \mathrm{C} \text { (faible) }\end{array}$ & Tohnishi et al., 2005 \\
\hline $\begin{array}{l}\text { Solubilité dans } \\
\text { certains solvants } \\
\text { organiques }\end{array}$ & $\begin{array}{c}\text { Méthanol : } 26,0 \mathrm{~g} / 1 \\
\text { Acétone }: 102 \mathrm{~g} / 1 \\
\text { n-heptane }: 0,0008 \mathrm{~g} / 1 \\
\text { Acétate d'éthyle : } 29,4 \mathrm{~g} / 1 \\
\text { 1-2 Dichloroéthane }: 8,12 \mathrm{~g} / 1\end{array}$ & APVMA, 2009 \\
\hline $\begin{array}{l}\text { Coefficient } \\
\text { d'adsorption, Koc }\end{array}$ & 1076- $3318 \mathrm{l} / \mathrm{kg}$ & \\
\hline $\begin{array}{l}\text { Coefficient de } \\
\text { partage octanol/eau } \\
\text { Kow }\end{array}$ & $\begin{array}{c}\text { Log Pow }=4,13 \pm 0,02 \mathrm{pH} 4 \\
\text { Log Pow }=4,14 \pm 0,02 \mathrm{pH} 7 \\
\text { Log Pow }=4,11 \pm 0,04 \mathrm{pH} 9 \\
\text { (liposoluble) }\end{array}$ & APVMA, 2009 \\
\hline DT50 eau $(\mathrm{j})$ & 15 à 39 & \multirow{6}{*}{ APVMA, 2009} \\
\hline DT50 sol (j) & 33,6 à 34,9 & \\
\hline Pression de vapeur & $<10^{-4} \mathrm{~Pa} 25^{\circ} \mathrm{C}$ & \\
\hline Point de fusion $\left({ }^{\circ} \mathrm{C}\right)$ & $217,5-220,7(99,6 \%$ pure $)$ & \\
\hline $\begin{array}{l}\text { Point d'ébullition } \\
\left({ }^{\circ} \mathrm{C}\right)\end{array}$ & $\begin{array}{l}\text { Non faisable en raison de la } \\
\text { dégradabilité thermique }\end{array}$ & \\
\hline $\begin{array}{l}\text { Constance de la loi } \\
\text { d'Henry }\end{array}$ & 2. $10^{-9} \mathrm{~Pa} . \mathrm{m}^{3} / \mathrm{mol}$ & \\
\hline
\end{tabular}


Tableau 4: Synthèse des caractéristiques physico-chimiques du spirotétramate.

\begin{tabular}{|c|c|c|}
\hline & Spirotétramate & Références \\
\hline Familles & Dérivées de l'acide tétramique & Bruck et al., 2009 \\
\hline Formule brute & $\mathrm{C}_{21} \mathrm{H}_{27} \mathrm{NO}_{5}$ & PRD, 2008 \\
\hline $\begin{array}{l}\text { Mode d'action sur les } \\
\text { insectes ciblés }\end{array}$ & $\begin{array}{l}\text { Inhibe la biosynthèse des lipides } \\
\text { chez les insectes ciblés }\end{array}$ & Nauen et al., 2006 \\
\hline Volatilité & Faible, stable & \multirow{11}{*}{ PRD, 2008} \\
\hline Hydro solubilité & $\begin{array}{l}33,5 \mathrm{mg} / \mathrm{l} \text { à } \mathrm{pH} 4 \\
29,9 \mathrm{mg} / \mathrm{l} \text { à } \mathrm{pH} 7 \\
19,1 \mathrm{mg} \text { à } \mathrm{pH} 9 \text { à } 20^{\circ} \mathrm{C}\end{array}$ & \\
\hline $\begin{array}{l}\text { Solubilité dans } \\
\text { certains solvants } \\
\text { organiques }\end{array}$ & $\begin{array}{l}\text { Ethanol : 44g/l à } 20^{\circ} \mathrm{C} \\
\text { Acétone : } 100-120 \mathrm{~g} / 1 \\
\text { Dichlorométhane : }>600 \mathrm{~g} / \mathrm{l} \\
\text { Acétate d'éthyle : } 67 \mathrm{~g} / 1 \\
\text { n-hexane } 0,055 \mathrm{~g} / 1 \\
\text { Toluène } 60 \mathrm{~g} / \mathrm{l} \\
\text { Diméthylsulfoxyde } 200-300 \mathrm{~g} / 1\end{array}$ & \\
\hline $\begin{array}{l}\text { Coefficient } \\
\text { d'adsorption Koc }\end{array}$ & - & \\
\hline $\begin{array}{l}\text { Coefficient de partage } \\
\text { octanol/eau Kow }\end{array}$ & $\begin{array}{l}\log K o e=2,51 \mathrm{pH} 4 \\
\log K o e=2,51 \mathrm{pH} 7 \\
\log K o e=2,50 \mathrm{pH} 9\end{array}$ & \\
\hline DT50 eau & Pas persistant & \\
\hline DT50 sol & Pas persistant & \\
\hline Pression de vapeur & $\begin{array}{l}\text { Valeurs extrapolées } \\
5,6.10^{-9} \mathrm{~Pa} \text { à } 20^{\circ} \mathrm{C} \\
1,5.10^{-6} \mathrm{~Pa} \text { à } 50^{\circ} \mathrm{C}\end{array}$ & \\
\hline Point de fusion & $142^{\circ} \mathrm{C}$ & \\
\hline Point d'ébullition & - & \\
\hline $\begin{array}{l}\text { Constance de la loi } \\
\text { d'Henry }\left(\mathrm{Pa} . \mathrm{m}^{3} / \mathrm{mol}\right)\end{array}$ & $6,99 \cdot 10^{-8}$ à pH 7 & \\
\hline
\end{tabular}

\section{Conclusion}

Il ressort de tout ce qui précède que le flubendiamide et le spirotétramate, des matières actives clées dans le traitement phytosanitaire du cotonnier au Bénin et dans la sous région ouest africaine ont des propriétés physico-chimiques et effets écotoxiques avérés sur les êtres vivants aussi bien terrestres qu'aquatiques. Il urge donc d'entreprendre, dans nos conditions d'utilisation de ces molécules, des études plus précises sur leurs impacts sur les écosystèmes en général, et ceux aquatiques, réceptacles finaux de ces biocides.

\section{REFERENCES}

Adégbidi A. 2000. Etude des Filières des Intrants Agricoles au Bénin: Engrais Minéraux Produits Phytosanitaires, Semences, Matériels et Equipements Agricoles, Fertilisants Organiques, (Tome 3). Presse de la FSA: Cotonou.

AIC (Association Interprofessionnelle de Coton). 2008. Plan de Campagne 
Agricole Cotonnière 2008-2009 et Perspectives 2009-2010. Presse de l'AIC: Cotonou.

AIC (Association Interprofessionnelle de Coton). 2009. Plan de Campagne Agricole Cotonnière 2009-2010 et Perspectives 2010-2011. Presse de l'AIC: Cotonou.

APVMA (Australian Pesticides and Veterinary Medecines Autority). 2009. Evaluation of the new active flubendiamide in the production BELT 480 SC insecticide and BELT $240 \mathrm{WG}$ insecticide. Commonweath of Australia Gazette No APVMA 2-3 février, Agricultural and veterinary chemicals code Act 1994, 1443-1335.

ARLA (Agence de Réglementation de la Lutte Antiparasitaire). 2004. Technical grade of spirotetramat: A subacute toxicity feeding study in the beagle dog. 201012 MRID: 469045-72, DACO: 4.3.2.

ARLA (Agence de Réglementation de la Lutte Antiparasitaire). 2005. Technical grade of spirotetramat. A subchronic toxicity testing study in the rat. 201136, MRID: 469045-38, DACO: 4.3.3.

ARLA (Agence de Réglementation de la Lutte Antiparasitaire). 2006a. 4-Methoxycyclohexanone, acute fish toxicity. 2006/0032/02, DACO: 9.9.

ARLA (Agence de Réglementation de la Lutte Antiparasitaire). 2006b. Acute toxicity of spirotetramat (tech.) to fish (Lepomis macrochirus). DOM 24056, MRID: 469044-39, DACO: 9.5.2.2.

ARLA (Agence de Réglementation de la Lutte Antiparasitaire). 2006c. Acute toxicity of spirotetramat (tech.) to fish (Oncorhynchus mykiss). DOM 24025, MRID: 469044-38, DACO: 9.5.2.1.

ARLA (Agence de Réglementation de la Lutte Antiparasitaire). 2006d. Early-life stage toxicity of spirotetramat (tech). to fish (Pimephales promelas). EBFN0305, MRID: 469044-42, DACO: 9.5.3.1.

ARLA (Agence de Réglementation de la Lutte Antiparasitaire). 2006e. Spirotetramat ecotoxicological equivalence assessment of the technical specification with the material tested in ecotoxicity studies. M274995-01-2, DACO: 2.13.3 CBI.

Bruck E, Elbert A, Fischer R, Krueger S, Kuhnhold J, Klueken AM, Ralf N, Jean-Francois N, Udo R, Hans-Juren S, Robert S, Xavier VW. 2009. Movento, an innovative ambimobile insecticide for sucking insect pest control in agriculture; biological profile and field performance. Crops Protection, 28: 838-844.

.Deguine JP, Ekukole G, Amiot E. 1993. La lutte étagée ciblée: un nouveau programme de protection insecticide en culture cotonnière au Cameroun. Coton Fibres Trop., 48: 99-119.

Ebbinghaus-Kintscher U, Luemmen P, Raming K, Masaki T, Yasokawa N. 2007. Flubendiamide, the first insecticide with a novel mode of action on insect ryanodine receptors. Planzenschutz-Nachrichten Bayer, 60: 117-140.

Fadoégnon B, Midingoyi S-KG. 2006. Référentiel Technico-économique de la Production Agricole: Comment Produire du Coton de Meilleure Qualité. Presse Locale: Cotonou.

Ferron P, Deguine JP, Moute JEM. 2006. Évolution de la protection phytosanitaire du cotonnier : un cas d'école. Cah. d'Agric., 15(1): 128-134.

Follin TC, Deat M. 1999. Le rôle des facteurs techniques dans l'accroissement des rendements en culture cotonnière. Coton et Développement Hors Série, 14-23.

Gomgnimbou APK, Savadogo PW, Nianogo AJ, Millogo-Rasolodimby J. 2009. Usage des intrants chimiques dans un agrosystème tropical: diagnostic du risque de pollution environnemental dans la région cotonnière de l'Est du Burkina Faso. Biotechnol. Agron. Soc. Environ., 13(4): 499-507.

Hall T. 2007. Ecological effects assessment of flubendiamide. PflanzenschutzNachrichtern Bayer, 60: 167-182. 
Haraguchi K, Kitamura T, Yamashuta T, Kido A. 1994. Simultaneous determination of traces pesticides in urban air. Atmosphere Environ., 28(7): 1319-1325.

IFDC (Centre International pour la Fertilité des sols et le Développement Agricole). 2007. Expériences d'apprentissage de la lutte etagée ciblée avec les producteurs à la base dans les conditions de banikoara. Rapport Technique d'Activités, Campagne 2006-2007. Presse Locale, Cotonou.

Justus K, Motoba K, Reiver H. 2007. Metabolism of flubendiamide in animals and plants. Pflanzenschutz-Nachrichtern Bayer, 60(2): 141-166.

Katary A. 2003. Etude spatio-temporelle de la gestion de la résistance de Helicoverpa armigera (Hubner, 1808) aux pyrethrinoïdes en culture cotonnière au Bénin. Thèse de Docteur d'Etat ès Sciences Naturelles, Université d'Abomey-Calavi, Cotonou, p.202.

MAEP (Ministère de l'Agriculture de l'Elevage et de la Pêche). 2005. Rapport annuel campagne agricole 2004-2005 au Bénin. Presse du ministère, p. 86.

Masaki T, Yasokawa N, Tohnishi M, Nishimatsu T, Tsubata $\mathrm{K}$, Inoue $\mathrm{K}$, Motoba K, Hirooka T. 2006. Flubendiamide, a Novel $\mathrm{Ca}^{+}$Channel Modulator, Reveals Evidence for Functional Cooperation between $\mathrm{Ca}^{+}$ Pumps and $\mathrm{Ca}^{+}$Release. Mol. Pharmacol., 69(5): 1733-1739.

Mbaye DF. 2008. Interdiction de l'Endosulfan dans les pays du Sahel en Afrique de l'Ouest. Rapport de consultation, groupe de travail PAN/IPEN sur les pesticides POPs. Presse Locale, Dakar.
MEF (Ministère de l'Economie et des Finances). 2010. Evaluation Ex-ante de la mise en œuvre des stratégies de relance du pôle coton-textile au Bénin. Rapport final. Presse du Ministère, Cotonou.

Nauen R, Bretschneider T, Elbert A, FischerR R, Reckmann U, Van Waetermeulen X. 2006. Biological and mechanistic considerations on the mode of action of spirotetramat. 11th IUPAC International Congress of Pesticide Chemistry, Aug 611. Book of Abstracts 2, Kobe, Japan, 109.

PRD (Projet de Décision d'Homologation) du spirotétramate. 2008. Publication par I'Agence de Réglementation de la lutte antiparasitaire de Santé Canada. p. 134.

Sattelle D, Cordova D, Cheek T. 2008. Insect ryanodine receptors: molecular targets for novel pest control chemicals. Invertebrate Neurosci., 8: 107-119.

Tohnishi M, Nakao H, Furuya T, Séo A, Kodama H, Tsubata K, Fujioka S, Hirooka T, Nishimatsu T. 2005. Flubendiamide, a novel insecticide highly active against Lepidopterous insect pests. J. Pestic. Sci., 30(4): 354-360.

Ton P. 1995. Le moteur blanc et le dévastateur blanc: coton et développement rural et dégradation des sols. In A la Recherche de l'Agriculture Durable au Bénin, Ton P, Dehan L (eds). Presse locale, Institut Voor, Université d'Amsterdam: Amsterdam.

Totin E. 2004. Libéralisation de la filière coton au Bénin. Thèse d'Ingénieur Agronome? Option Economie socioanthropologie communication, FSA/ Université d'Abomey-Calavi (Bénin), p.187. 